\title{
Quantitative Phase Shift Variance Imaging
}

\author{
M Siepmann ${ }^{1}, \mathrm{~J} \mathrm{Bzyl}^{2}$, S Fokong ${ }^{2}$, F Kiessling ${ }^{2}$, G Schmitz ${ }^{1}$ \\ ${ }^{1}$ Department of Medical Engineering, Ruhr-University Bochum, Germany, Georg.Schmitz@rub.de \\ ${ }^{2}$ Department of Experimental Molecular Imaging (ExMI), Medical Faculty, RWTH Aachen, Germany
}

\begin{abstract}
Molecular ultrasound imaging is based on the detection of contrast agents (microbubbles) targeted to disease specific receptors in the vasculature. By quantifying the amount of the adherent microbubbles a change in the expression of these receptors and thus the disease development can be assessed. Quantification of microbubbles from ultrasound images in vivo was thus far mainly performed by analysis of the image intensity. In this work, the approach of imaging and counting single microbubbles is pursued. Detection of microbubbles is performed with the Phase Shift Variance Imaging technique recently developed in our research group. The detection of single microbubbles is first verified with microbubbles embedded in gelatine phantoms. Subsequently images from microbubbles targeted to VEGFR-2 are acquired from mouse tumor models to validate the method in vivo. The results show that single bubbles can be detected with a contrast-to-background ratio of $50 \mathrm{~dB}$ in the phantoms. Concentration estimates show that absolute quantification is possible with the proposed method. Bubble detection could also be verified in vivo; however minor motion and flow artefacts are present in the pre contrast scans.
\end{abstract}

\section{Introduction}

Within the last decade ultrasound has gained attention in the field of molecular imaging. Here, the expression of molecular disease-associated markers is visualized by imaging target-bound specific ultrasound contrast agents. These commonly consist of encapsulated gasmicrobubbles which are injected into the vasculature. Upon contact with disease specific receptors at the endothelium they are retained and thus indicate the presence and location of the target molecules.

In oncology, a primary goal of ultrasound molecular in vivo imaging is the assessment of tumor angiogenesis, i.e. the formation of new blood vessels. A further important aim is the assessment of therapy response with regard to an altered tumor-associated marker expression. This allows the evaluation of the efficacy of novel drug candidates as well as treatment monitoring in patients. The detection of such treatment effects requires quantification of the contrast agent, which is nowadays mainly based on the evaluation of image intensity allowing only a relative quantification usually in a 2-dimensional slice. Here, we investigate phantoms and in vivo the feasibility of absolute quantification assessing the complete tumor volume.

A 3-dimensional technique for quantifying single microbubbles (MB) was developed by Reinhardt et al [1]. The method is based on the detection of MB destruction events and was named Sensitive Particle Acoustic Quantification (SPAQ). In general, the number of microbubbles (MB) detected within one frame is too high to allow the separation of individual MB. Reinhardt et al. therefore artificially reduced the imaging volume by destroying all bubbles within one slice and subsequently moving the transducer in elevational direction. Destruction then occurs only within the new volume determined by the motion step size. The events may then be counted and yield a concentration estimate.

Reinhardt et al. used Doppler techniques for the detection of destruction events. However, Doppler imaging cannot differentiate between bubble destruction and blood flow. Recently, we developed a new method for destructive MB imaging based on the variance of the phase shift between consecutive ultrasound pulse echoes, to avoid this ambiguity [2]. The single bubble detection capability of this technique is now validated with a high frequency small animal imaging device.

The SPAQ method was tested by Reinhardt et al. in phantom and ex vivo experiments. In this work we will apply the method in vivo in combination with our proposed detection technique. A primary goal of molecular in vivo imaging is the assessment of tumor angiogenesis, the formation of new blood vessels. Therefore, in this work the detection of microbubbles targeted to the angiogenic marker Vascular Endothelial Growth Factor Receptor 2 (VEGFR-2) is investigated in epidermoid cancer xenografts in nude mice.

\section{Methods}

The Phase Shift Variance (PSV) technique is employed for the detection of single bubble destruction events. The principle of the PSV method was described in [2] and is briefly reviewed in section 2.1 together with a description 
of the data acquisition. First, it is tested whether absolute quantification can be performed with the proposed detection method, indicating sensitivity to single destruction events. Then in vivo experiments with tumor bearing animals are performed.

\subsection{Data Acquisition and Processing}

The PSV technique requires the acquisition of echoes from a sequence of identical ultrasound pulses, like standard Doppler methods. It is well known that destruction events are visualized in Doppler as they cause changes in the phase shift $[3,4]$. However, to differentiate bubbles from motion, here, the variance of the phase shift is used as a detection criterion instead of the phase shift itself [2].

Assuming linear motion, the variance of the phase shift $\sigma_{\Delta \theta_{k}}^{2}$ depends on the signal to noise ratio SNR, which is estimated from the signal amplitude $r$ by

$$
\sigma_{\Delta \theta_{k}}^{2}=\frac{1}{\mathrm{SNR}_{i}}+\frac{1}{\mathrm{SNR}_{i+1}}, \quad \hat{\mathrm{SNR}}_{i}=\frac{r_{i}^{2}}{\sigma_{N}^{2}}-2,(1)
$$

where $i$ denotes the pulse index, $k$ the index of the phase shift and $\sigma_{N}^{2}$ the variance of the measurement noise. Normalizing the phase shift by the respective variance $\sigma_{\Delta \theta}{ }^{2}$ yields a set of approximately standard Gaussian distributed variables. The PSV can then be then calculated from $K$ phase shifts by

$$
P S V=\frac{1}{K-1} \sum_{k=1}^{K}\left(\frac{\Delta \theta_{k}-\overline{\Delta \theta}}{\sigma_{\Delta \theta_{k}}}\right)^{2}
$$

This PSV approximately follows a chi-square distribution. A deviation from this indicates the presence of contrast agent and is used for detection. The system used in this study is sensitive enough to detect vibrations in the building. As these vibrations deviate from the linear model used in [2] an additional vibration term $\sigma_{V}^{2}$ was included in the calculation of the variance

$$
\sigma_{\Delta \theta}^{2}=\frac{1}{S N R_{i}}+\frac{1}{S N R_{i+1}}+\sigma_{V}^{2}
$$

This term is obtained by calculating the variance of the weighted average phase shift across the whole image depth.

The PSV detection method was used in combination with the SPAQ technique [1] for quantification. All acquisitions were performed with a dedicated small animal imaging system Vevo 2100 (Visualsonics, Toronto, Canada). Images were recorded in Power Doppler mode, storing the digitized In-phase/Quadrature (IQ) data for offline processing. A transducer with $21 \mathrm{MHz}$ center frequency was used, transmitting pulses at a pulse repetition frequency of $1 \mathrm{kHz}$. The system offers a 3D mode in which the transducer is moved in elevational direction after the acquisition of each frame. Thus the SPAQ can be directly applied by using the 3D Power Doppler mode of the Vevo 2100.

The phase shift variance was calculated offline in MATLAB (Mathworks, Natick, MA, USA) as described above. 16 pulses per line were used for calculation of the variance. The quantification was performed according to the statistical formula derived in[5].

\subsection{Phantom Experiments}

Gelatine phantoms were produced containing different MB concentrations and silica gel as background scatterers. Therefore, $5 \mathrm{~g}$ of cold soluble gelatine were dispersed in $100 \mathrm{ml}$ water. The suspension was refrigerated until it became semi-fluid. Contrast agent was then added under constant stirring together with $0.2 \mathrm{~g}$ of silica gel. The phantoms were then again refrigerated until solid. The MB concentration in the original suspension was quantified with a Coulter Multisizer 3 (Beckman Coulter) and diluted in the gelatine to yield phantom concentrations of $10^{3}$, $5 \cdot 10^{3}$ and $10^{4} \mathrm{MB} / \mathrm{ml} .3$ Phantoms were prepared for each concentration and an additional phantom without $\mathrm{MB}$ was prepared identically. SPAQ acquisitions of these phantoms were acquired as described above. The step size was varied from 51 to $299 \mu \mathrm{m}$. Each volume was scanned repeatedly for 3 times.

The average spot size is calculated from another gelatine phantom with $10^{3} \mathrm{MB} / \mathrm{ml}$ prepared as described above. Microbubbles are detected and counted, yielding the MB concentration in the acquired volume.

\subsection{Animal Experiments}

For in vivo tests, human epidermoid cancer xenografts were induced in two nude mice. Therefore, $4 \cdot 10^{6}$ A431 cells were injected subcutaneously into the hind limb of two CD-1 mice (Charles River). Tumors were grown to a size of approximately $4 \mathrm{~mm}$ diameter.VEGFR-2 targeted MBs were injected intravenously and allowed to adhere for several minutes. Ultrasound imaging was performed as described above for the gelatine phantoms. A SPAQ step size of $101.6 \mu \mathrm{m}$ was selected. A baseline scan was also recorded prior to contrast agent administration for both animals. Two types of contrast agent were used for the in vivo tests: The commercial contrast agent Micromarker, which is encapsulated by a lipid shell, and an experimental poly n-butyl cyanoacrylate (PBCA) shelled agent, which is described in [6].

\section{Results}

\subsection{Phantom Experiments}

Image 1 shows PSV images from gelatine phantoms containing no (a) and $5 \cdot 10^{3} \mathrm{MB} / \mathrm{ml}$ (b). The phantom results show that single bubbles are enhanced by up to 50 $\mathrm{dB}$ compared to background. No events are detectable in 
a)

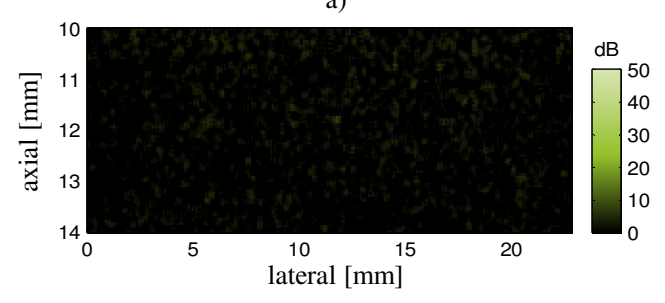

b)

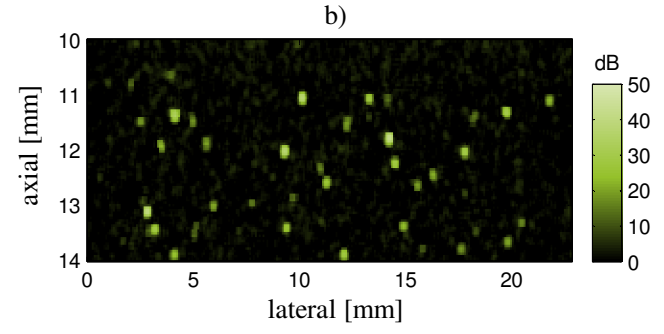

Image 1 Phase Shift Variance Images: a) empty phantom, b) phantom with $5 \cdot 10^{3} \mathrm{MB} / \mathrm{ml}$

the phantom without MB. The destruction of bubbles is restricted to a region around the focus of the transducer, which is evaluated for the concentration estimates. The measured concentrations are shown in Image 2. For the lower concentrations the calculated number of MB deviates by less than $10 \%$ from the actual concentration. At $10^{4} \mathrm{MB} / \mathrm{ml}$ a slight underestimation of the concentration is observed. No trend can be seen of the estimated concentration with step size.

\subsection{Animal Experiments}

Image 3 shows images from the two analyzed tumors. On the left, the b-mode image is displayed with the tumor contour outlined. The middle and right hand images show the scans before and after contrast agent administration, respectively. A $2 \times 2$ median filter was applied to further reduce noise. Microbubble destruction events are only visible in the post injection scans.

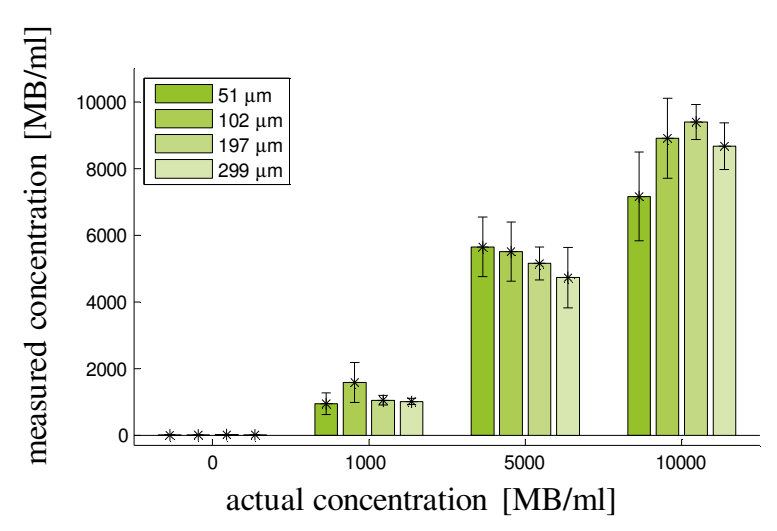

Image 2 Calculated concentrations. Error bars show the standard deviation over three phantoms.
From the color pixel densities the concentration was calculated as it was done for the phantom experiments. The concentration estimates yielded results of $5.7 \cdot 10^{3} \mathrm{MB} / \mathrm{ml}$ for the PBCA bubbles and $12.3 \cdot 10^{3} \mathrm{MB} / \mathrm{ml}$ for Micromarker bubbles. In the pre-scans $1.20 \cdot 10^{3}$ and $1.27 \cdot 10^{3} \mathrm{MB} / \mathrm{ml}$ Micromarker and PBCA bubbles are detected respectively, indicating the presence of artefacts.

These artefacts occurred in regions of a larger blood vessel or at the high intensity reflection at the animal skin. The vessel artefacts persisted over several frames and were visible in repeated acquisitions. An overview of the distribution of detected agent in 3D is shown in Image 4. Here the isosurface of the PSV is shown at a threshold of $15 \mathrm{~dB}$ for the Micromarker bubbles.

\section{Discussion and Conclusion}

It was demonstrated that the proposed technique allows the absolute quantification of MB concentrations in vitro and in vivo. No bubbles are visible in the empty phantoms, while spots with maxima up to $50 \mathrm{~dB}$ are caused by MB.

The method relies on the destruction of the MB. The zone of destruction is however restricted to the focus of the transducer. Thus concentration estimates are only accurate within this region. The estimates are in good agreement, although a slight underestimation towards higher concentrations can be seen. Not all bubbles above the analyzed region are destroyed, due to the restriction of destruction to the focal zone. The underestimation for higher concentration is therefore most likely due to acoustic shadowing, which prevents a complete destruction of the bubbles. These effects could be reduced by using multiple transmit foci.

The acquisition was performed using the Doppler mode of the system. This implies that the time for acquisition and achievable frame rates are in the range of that of standard Doppler techniques. Furthermore it shows that standard hardware can be used and no modifications to existing systems need to be made. Therefore the technique is applicable with any commercial system. A 3D acquisition however is necessary for quantification. This requires a translational stage which is synchronized with the image acquisition, or electronic steering with a $2 \mathrm{D}$ array.

While no artefacts were present in the phantoms, some motion artefacts occurred in the in vivo images. As mentioned initially, the model is only valid for linear motion with an additional vibration component. It is evident that this presents only an approximation to the motion in vivo. The flow artefacts occurred repeatedly and may thus be eliminated by baseline scans.

The technique was tested with a lipid and a polymer shelled MB type. It is known from literature that these have different mechanisms of destruction [7]. Both types 
of agent were detectable, indicating that the PSV is sensitive to different ways of bubble disintegration.

In conclusion, it was demonstrated that absolute quantification is feasible with the proposed method. Future studies will assess therapy outcome with this technique.

\section{Acknowledgement}

This work is funded by the German Research Foundation (DFG) Project Number SCHM 1171/3-1.

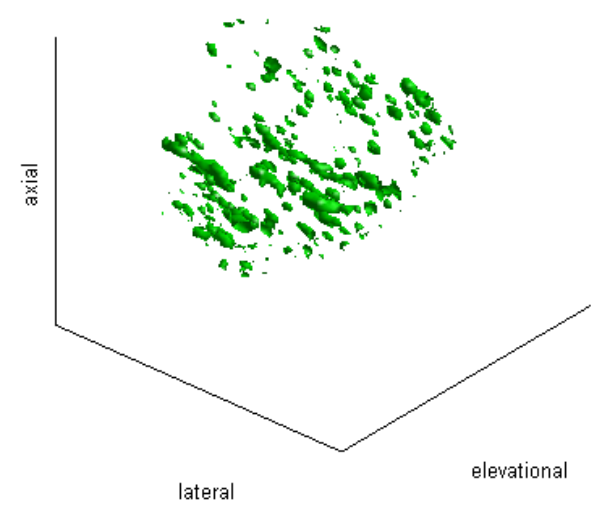

Image 4 3D volume of tumor with adherent Mikromarker bubbles. The threshold for the PSV was set to $15 \mathrm{~dB}$.

\section{References}

[1]M. Reinhardt, P. Hauff, A. Briel, V. Uhlendorf, R. A. Linker, M. Maeurer, M. Schirner, Sensitive particle acoustic quantification (SPAQ) a new ultrasound-based approach for the quantification of ultrasound contrast media in high concentrations, Invest Radiol 40 (2005)2-7.

[2]M. Siepmann, M. P. Mienkina, S. Fokong, Z. Liu, J. Gätjens, F. Kiessling, S. G., Phase shift variance imaging for contrast agent detection, Proc IEEE Ultras Symp 2010 (2010) 1944 - 1947.

[3]M. Ressner, T. Jansson, J. Cedefamn, P. Ask, B. Janerot-Sjoberg, Contrast biases the autocorrelation phase shift estimation in doppler tissue imaging, Ultrasound Med Biol 35 (3) (2009) 447-457.

[4]K. Tiemann, C. Pohl, T. Schlosser, J. Goenechea, M. Bruce, C. Veltmann, S. Kuntz, M. Bangard, H. Becher, Stimulated acoustic emission: Pseudo-doppler shifts seen during the destruction of nonmoving microbubbles, Ultrasound Med Biol 26 (7) (2000) 1161-1167.

[5]M. Siepmann, M. Reinhardt, G. Schmitz, A statistical model for the quantification of microbubbles in destructive imaging, Invest Radiol 45 (10) (2010) 592-599.

[6]S. Fokong, M. Siepmann, Z. Liu, G. Schmitz, F. Kiessling, J. Gätjens, Advanced characterization and refinement of poly n-butyl cyanoacrylate microbubbles for ultrasound imaging, Ultrasound Med Biol 37 (10) (2011) 1622-1634.

[7]S. H. Bloch, M. Wan, P. A. Dayton, K. W. Ferrara, Optical observation of lipid- and polymer-shelled ultrasound microbubble contrast agents, Appl Phys Lett84 (4) (2004) 631-633.
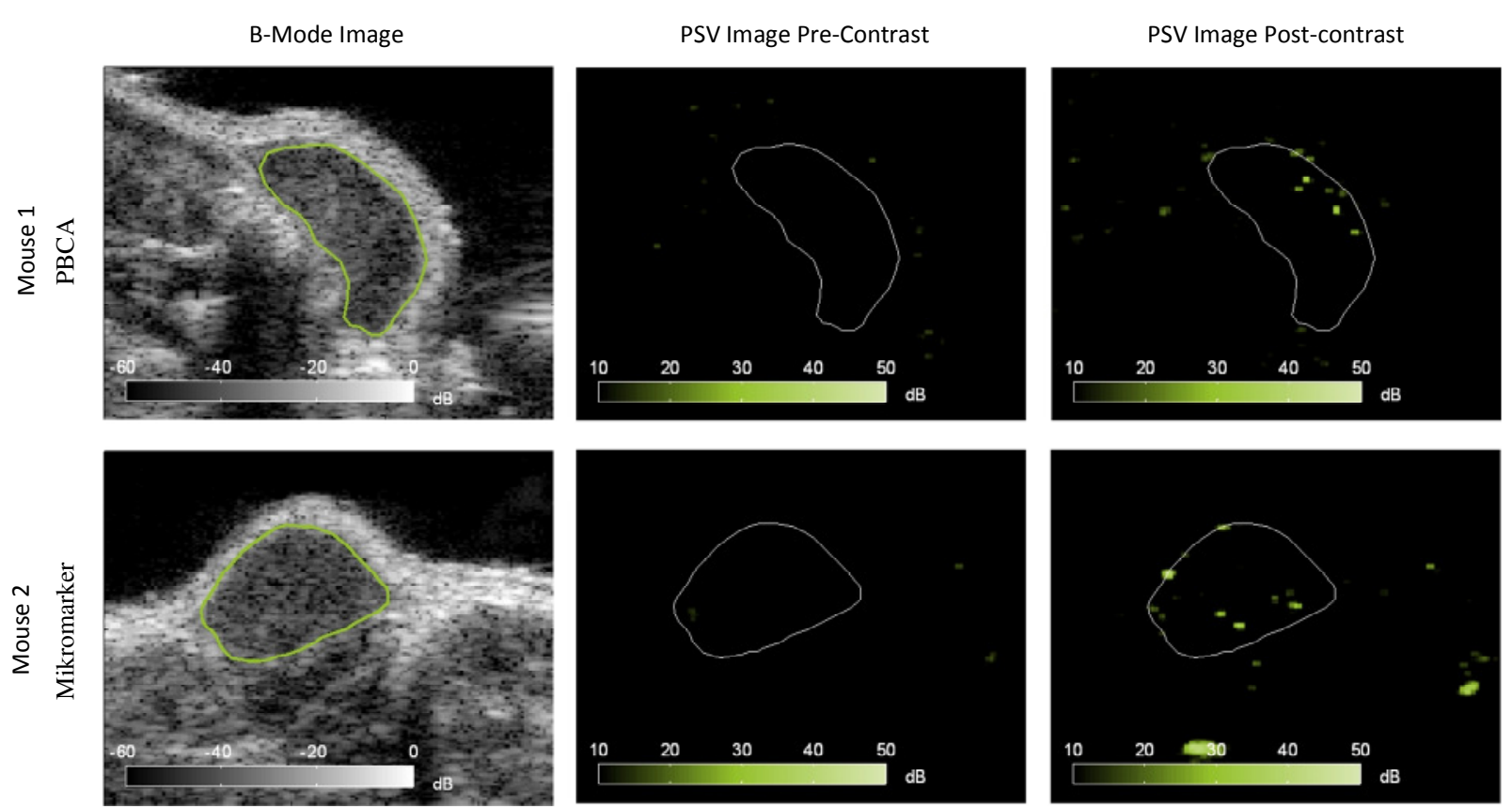

Image 3 Frames from in vivo acquisitions: The left panes show the b-mode images with the tumor contour outlined. In the middle the pre-contrast scans are displayed in comparison to the post-contrast images on the right. In the latter, contrast agent is clearly visible. 